\title{
AGE, SEX, AND SETTING IN THE ETIOLOGY OF STROKE STUDY (ASSESS)
}

Mahmoud Reza Azarpazhooh ${ }^{1,2,3}$, Amanda G. Thrift ${ }^{4}$, Luciano A. Sposato ${ }^{2,3,5,6}$, Negar Morovatdar ${ }^{7}$, Amin Amiri $^{1}$, M Cecilia Bahit ${ }^{8}$, Mariano Coppola ${ }^{9}$, Jennifer L. Mandzia ${ }^{2}$, Nawaf Yassi ${ }^{10}$, Yongchai Nilanont ${ }^{11}$, Antonia Nucera ${ }^{12}$, Subhash Kaul ${ }^{13}$, Suvarna Alladi ${ }^{14}$, Moira K. Kapral ${ }^{15}$, Brian Silver ${ }^{16}$, David Werring ${ }^{17}$, Robert Simister ${ }^{18}$, Richard H. Swartz ${ }^{19}$, Mayowa Ojo OwolabiI ${ }^{20}$, Bruce Ovbiagele ${ }^{21}$ and Vladimir Hachinski ${ }^{2,3}$ on behalf of ASSESS investigators

${ }^{1}$ Department of Neurology, Ghaem hospital, Mashhad University of Medical Sciences, Mashhad, Iran.

${ }^{2}$ Department of Clinical Neurological Science, University Hospital, London Health Sciences Center, Western University, ON, Canada.

${ }^{3}$ Department of Epidemiology and Biostatistics, Western University, ON, Canada.

${ }^{4}$ Stroke and Ageing Research, Department of Medicine, School of Clinical Sciences at Monash Health, Monash University, Clayton, Victoria, Australia.

${ }^{5}$ Department of Anatomy and Cell Biology, Western University, ON, Canada.

${ }^{6}$ Stroke, Dementia, and Heart Disease Lab, Western University, ON, Canada.

${ }^{7}$ Clinical Research Unit, Mashhad University of Medical Sciences, Mashhad, Iran.

${ }^{8}$ Cardiology Department, INECO Neurociencias Oroño, Rosario, Santa Fe, Argentina.

${ }^{9}$ Hospital Ramon, Santamarin, Tandil, Argentina.

${ }^{10}$ Departments of Medicine and Neurology, Royal Melbourne Hospital University of Melbourne, Parkville, Australia.

${ }^{11}$ Department of Medicine, Siriraj Hospital, Mahidol University, Bangkok, Thailand.

${ }^{12}$ Department of Neurology, Saint Andrea Hospital, Stroke Unit, La Spezia, Italy.

${ }^{13}$ Department of Neurology, Nizam's Institute of Medical Sciences, Hyderabad, India.

14 National Institute of Mental Health and Neurosciences, Bengaluru, India.

${ }^{15}$ Department of Medicine and Institute of Health Policy, Management and Evaluation, University of Toronto, Toronto, Canada.

${ }^{16}$ Department of Neurology, The University of Massachusetts Medical School and UMass Memorial Medical Center,Worcester, Massachusetts, United States.

${ }^{17}$ Stroke Research Centre, UCL Institute of Neurology, London, United Kingdom.

${ }^{18}$ University College London Hospitals, NHS Foundation Trust, London, United Kingdom.

19 Department of Medicine, Division of Neurology, Sunnybrook HSC, University of Toronto, Toronto 
Canada.

${ }^{20}$ Center for Genomic and Precision Medicine, University of Ibadan, Nigeria.

${ }^{21}$ Department of Neurology, Medical University of South Carolina, USA.

Corresponding author:

Vladimir Hachinski, MD, FRCPC, DSc

339 Windermere Road, London, Ontario, Canada

Tel: 519-663-3624

Email: Vladimir.hachinski@1hsc.on.ca

Cover title: ASSESS: A comparison among three cohorts from Argentina, Australia and Iran

Keywords: Stroke, etiology, population-based data, age, epidemiology, multicenter studies.

Word count: 5959

Number of tables: 2

Number of figures: 4

Number of supplementary files: 7 


\section{Abstract}

Background and Purpose: Little is known about variations in stroke etiology in different world regions.

Methods: Age, Sex, and Setting in the Etiology of Stroke Study (ASSESS) is an ongoing multicenter study from different geographic areas worldwide. Data from all centers will be categorized according to age, sex, setting, stroke subtypes and where available, cognitive status.

Results: So far, 12 centers in the world have joined ASSESS. In this report, we harmonized and compared all available data on stroke demographics, risk factors, and subtypes from centers with population-based data in Argentina, Australia, and Iran. In total, 2274 patients with first-ever stroke were recruited. Women were significantly older than men at the time of stroke in Argentina (74.1 \pm 15.6 years vs 67.5 $\pm 13.6 ; \mathrm{p}<0.001)$ and Australia $(75.8 \pm 15$ years vs $71.5 \pm 13.4$ years; $\mathrm{p}<0.001)$ but not in Iran $(64.1 \pm 15.3$ years vs $64.9 \pm 14.6$ years; $\mathrm{p}=0.5)$. The largest age-adjusted incidence rates (per 100,000/year) were observed in Iran (233.8) followed by Australia (100.5) and Argentina (87.4). Stroke of undetermined source was the most frequent subtype in all studies. After this subtype, the majority of strokes in Australia were embolic, while small artery disease was more frequent in Argentina and Iran. Risk factor profiles were different, with hypertension and diabetes being more prevalent in Argentina and Iran, while atrial fibrillation, transient ischemic attacks, and myocardial infarction were more common in Australia.

Conclusions: ASSESS will enable comparisons of data from different regions to determine the age and sex distribution of the most common causes of stroke in each setting. This will empower health policy makers to design preventive measures by targeting the specific characteristics of each population. According to our first report, while in low-middle income countries more resources should be allocated 
to control traditional risk factors, such as hypertension and diabetes, the identification of atrial fibrillation and prevention of embolic stroke are crucial in high-income countries. 


\section{Introduction}

Highly dynamic and variable trends of major diseases in the world require large collaborative national/ international studies. In recent decades there has been a substantial decrease in the incidence and mortality rate of stroke in high-income countries, but this has not been observed in low- to middleincome countries, where the majority of strokes occur. (1) Such temporal trends in stroke incidence may vary with factors such as global changes in socioeconomic status, environmental factors (air pollution) and aging populations and therefore ongoing monitoring is required. However, there are multiple obstacles to monitoring, including the need for international collaboration, scarce stroke information at a population level in many countries, and challenges in pooling and comparing data due to lack of harmonized data collection processes. This major need in world health data is fulfilled partly by the Global Burden of Disease (GBD) study, providing important worldwide trends in several diseases including stroke. (1) However, the results of many countries are based not on data but on sophisticated estimations. (2)

The Age, Sex, and Settings in the Etiology of Stroke Study (ASSESS) was designed as a multicenter study of stroke to systematically gather and monitor data from different countries using a highly flexible approach. In the current paper, we report the study design, rationale, and methods, and we invite researchers worldwide to join this collaborative initiative. In addition, we summarize stroke incidence rates, stroke subtypes and associated vascular risk factors based on the analysis of collaborating centers with population-based data, namely Argentina, Australia, and Iran.

\section{Methods:}

\section{Ethics:}

ASSESS is an ongoing multicenter stroke study. All centers should provide their Ethics Committees 
approval. We anticipate that participating centres will share anonymized data, which will be secured in protected servers and will remain confidential in an encrypted and secured dataset (RedCap), according to the requirements of the Lawson Research Institute, London, ON, Canada.

\section{Data management and plan:}

We plan to investigate variations in stroke incidence, risk factors, quality of care (preventive strategies and acute stroke care), and short- and long-term outcomes. We also invite researchers to propose reports addressing local, regional, global knowledge gaps about stroke. An international steering committee will be created with the purpose of analyzing research proposals and to guide future steps. We also invite young investigators to lead specific research projects under the guidance of senior scientists at each centre. Collaborators will be considered as co-authors of every publication if they meet the definition of author by the International Committee of Medical Journal.

Data from studies in all centers will be harmonized and categorized according to whether the study was hospital- or population-based. We have developed a baseline list of variables with their definitions to facilitate the process of data-sharing to researchers interested in collaborating. We acknowledge that data across studies is not homogeneous and we do not expect other datasets to perfectly match the needs of this study. ASSESS will have a flexible policy for data sharing and analysis. We can either analyze at the coordinating centre (London, ON, Canada) or we can receive the results after being analyzed at each participating center.

\section{Summary of studies}


Twelve stroke centers in different regions of the world (Figure 1) have agreed to participate in this study:

\section{The Program for the Epidemiological Evaluation of Stroke in Tandil, Argentina (PrEViSTA)}

PrEViSTA, is a prospective population-based study of first-ever stroke and transient ischemic attack (TIA) in Tandil, Argentina, South America. It was conducted between January 5, 2013, and April 30, 2015. Using several overlapping strategies, all cases of first-ever strokes were identified. Overall, 334 patients with FES were recognized and were followed for 12 months after their index stroke. (3)

\section{The North East Melbourne Stroke Incidence Study (NEMESIS)}

The NEMESIS is a large population-based study of stroke in Australia. The study was performed in two consecutive phases. In brief, from May 1, 1996 to April 30, 1997 in a defined area of northeast Melbourne (133,816 residents) all suspected cases with stroke were identified ( $\mathrm{n}=353$ individuals, with 281 being first-ever strokes). (4) In the second phase (between May 1, 1997, and April 30, 1999) and after the expansion of the catchment areas to 22 post codes (population 306,631), 1035 patients with first-ever stroke were recruited. (5) Patients were followed for 10 years after the index stroke.

\section{The Mashhad Stroke Incidence Study (MSIS)}

The MSIS is a large prospective population-based stroke study in Mashhad, northeast Iran, Middle East. Between 21 November 2006 and 20 November 2007, using multiple overlapping sources and with help from almost 1000 community health volunteers, a door to door survey was performed in three distinct 
regions of Mashhad (population: 450,229). Both hospital (admission, outpatients) and non-hospital (death certificates, private clinics, specialist referrals and national datasets) sources were used to identify any potential cases with stroke. 684 patients with acute stroke (624 with first ever stroke) and were followed for the next 5 years after their index event (6,7) (online Supplementary table I; please see http://stroke.ahajournals.org).

\section{The Royal Melbourne Hospital Stroke Registry}

The Royal Melbourne Hospital is a major public teaching hospital in metropolitan Melbourne, Australia. The stroke unit treats approximately 800 patients per year with ischemic or hemorrhagic stroke, including approximately 100 intravenous thrombolysis cases. The hospital is also a 24-7 statewide referral centre for endovascular thrombectomy for the state of Victoria (population 6 million), and performs approximately 200 endovascular thrombectomy cases per year. A prospective stroke clinical database has been collected since 2001 .

\section{The Ontario Stroke Registry}

The Ontario Stroke Registry (OSR) comprises data on all consecutive patients with acute stroke or TIA seen in the emergency department or admitted to any of 11 regional stroke centres in the province of Ontario, Canada, North America, as well on a population-based sample of patients with stroke/TIA seen at every acute care hospital across the province between 2003 and 2013. The registry, with a sample size of $>90,000$, is housed at the Institute for Clinical Evaluative Sciences (ICES) where it is linked to population-based administrative databases using unique, encoded identifiers to provide indefinite follow up for mortality, readmissions, physicians services and other outcomes, as well as 
information on medication use, area of residence and other demographic variables.

\section{The Hyderabad Stroke Registry}

The Hyderabad Stroke Registry is an ongoing, prospective hospital-based observational project based in Nizam's Institute of Medical Sciences, Hyderabad, the capital city of Telangana, South India, designed to comprehensively evaluate and record clinical, radiological, laboratory and outcome data of consecutive patients with acute stroke. (8) Nizam's Institute of Medical Sciences is a major neurological centre and teaching hospital in the city of Hyderabad with a large catchment area for neurological disorders. Patients are referred by practicing physicians or can register themselves. The patient population is fairly representative of the disease pattern in this region.

\section{The La Spezia Stroke Registry}

The La Spezia Stroke Registry is an ongoing hospital-based survey in the Stroke Unit, Sant Andrea Hospital, a community hospital in La Spezia, Italy, Europe. La Spezia is a port city in Italy with a catchment area of 225,000 residents. All information on patients with stroke admitted in this center has been recorded since January 2014.

\section{The Siriraj Stroke Registry}

The Siriraj Stroke Registry is a large prospective hospital-based study in the Siriraj Stroke Center, Siriraj Hospital Bangkok, Thailand, Southeast Asia. Overall, 2,942 patients with acute stroke were registered between 2007 and 2010. 


\section{The University of Massachusetts Medical School and UMass Memorial Medical Center Stroke}

\section{Registry}

The University of Massachusetts Medical School and UMass Memorial Medical Center Stroke Registry is an ongoing hospital-based survey in Worcester, Massachusetts, United States. Worcester County is in central Massachusetts and the university hospital serves a catchment area of over 800,000 residents. All information on patients with stroke have been collected since June 2017.

\section{Screening of Depression, Obstructive Sleep Apnea and Cognitive Impairment (DOC Utility study)}

The "DOC Utility" study is a prospective study funded by the Canadian Institute of Health Research to assess the impact of Depression, Obstructive sleep apnea and Cognitive impairment (DOC) symptoms on long-term outcomes in patients from stroke prevention clinics. We are prospectively screening over 7,000 people at baseline visits to clinics across 8 regional stroke prevention clinics in Ontario, Canada. The outcomes will be 1-year event rates of recurrent stroke, heart attack, admission to long-term or complex continuing care and death, as defined by population-based administrative health records.

\section{Stroke Investigation in North And central London (SIGNAL study)}

The study is an ongoing clinical stroke registry in London, the United Kingdom incorporating detailed stroke phenotyping (including advanced early MRI) embedded into standard clinical care, with follow up for clinical events and functional status. Currently over 1000 patients have been included, and it is expected that over 1000 patients with stroke will be included annually over the next 5 years.

\section{The Stroke Investigative Research and Education Network (SIREN)}


The Stroke Investigative Research and Education Network (SIREN) (9-12) has the largest population of Africans with stroke in a single study comprising about 4000 cases and 4000 controls from many sites in Ghana and Nigeria, accurately phenotyped with state-of-the-art tools (pictographic questionnaire for stroke-free status for controls, and AIM-on-Clear Canvas Stroke phenotyping Software tool for cases). (13) Subjects are comprehensively evaluated with a set of traditional and novel socio-demographic, clinical, and biochemical. Furthermore, the controls, who are recruited from the same catchment population as the cases are matched for age, sex and ethnicity. SIREN is aimed at unravelling the genetic and environmental risk factors for stroke among Africans and has identified the dominant environmental risk factors for stroke on the continent. SIREN, which commenced in 2013 is part of the H3Africa consortium. (9-12)

\section{Statistical analyses:}

In the current report, we calculated the age-adjusted stroke incidence rates per 100,000 and evaluated stroke subtype in patients with first-ever strokes from the three population-based studies included in ASSESS. Using the direct method, incidence rates were age-standardized to the new world standard population (WHO 2000-2025). We used Chi Square test to determine differences in the frequency of risk factors and demographic data among the three centers. Age distribution was tested via the Mann Whitney U test or Kruskal Wallis test. We used the statistical process control (SPC) to predict the frequency of different stroke subtypes according to the patient's age. The SPC is used to recognize structural changes in a process. (14) It includes a control chart with three lines: the center line, the upper and lower control limit ( \pm 3 sigma). In the SPC, a process is defined as "stable", "in-control" or "predictable" when data are within the control limits; i.e. between the upper and lower limits. In the current study, we determined instability using the following two rules: (1) "moving range of control rules" three sigma above the central line; and (2) "moving range of control rules" two out of the last 
three points higher than two sigma. Data distribution in the control limits represents expected frequency at age points in which a stroke subtype might occur. Instability points out of predicted ranges were described as variation of special causes. (15) Natural variations (variations within the control limits), that would be expected in any quality process assessment, were defined as variation of common causes. We used the XMR (Individual Moving Range) chart to determine instability point of age for different stroke subtypes. A p-value $\leq 0.05$ (two sided) was considered to be statistically significant. The SPSS (version 16, SPSS Inc., IL, and USA) and R (version 3.2.5) statistical software were used for all statistical analyses.

\section{Results:}

\section{Characteristics of patients}

In total, 2274 patients with first-ever stroke, including 334 in the PrEViSTA (female: 52.4\%), 1316 in the NEMESIS (female: 55.5\%), and 624 in the MSIS (female: 47.6\%) were recruited in 3 separate cohorts. A large proportion of patients were imaged (CT/MRI; 79.3\% PrEViSTA, 89.7\% NEMESIS, 97.9\% MSIS). Stroke frequency according to age was normally distributed in the MSIS with the highest rate in those aged between 60 and 75 years for both sexes. In Argentina and Australia, the stroke frequency increased in patients aged 70 years and above, particularly among women (Figure 2).

In the MSIS, no significant sex difference was observed according to participants age at the time of stroke. However, women were significantly older than men in the NEMESIS ( $75.8 \pm 15$ vs $71.5 \pm 13.4$; 
$\mathrm{p}<0.001)$ and the PrEViSTA study $(74.1 \pm 15.6,67.5 \pm 13.6 ; \mathrm{p}<0.001)$. In general, patients with stroke in Iran were significantly younger than patients in Australia and Argentina (Table 1). A significant male predominance was observed in the MSIS participants as compared to their counterparts (PrEViSTA: 47.60\%; NEMESIS: 44.50\%; MSIS: 52.40\%; p=0.005).

\section{Age-adjusted incidence rate}

The age-adjusted incidence of stroke according to the WHO world population was greater in Iran (233.8 per 100,000/year) than Australia (100.5) and Argentina (87.4). Although ischemic stroke had the highest age-adjusted incidence rate among all types of stroke, an obvious difference was observed between the centers (Table 2). In those aged $<55$ years the incidence of ischemic stroke adjusted to the WHO world population was 12.8 per 100,000/year in PrEViSTA, lowest in NEMESIS (9.1) and greatest in MSIS (30.1). For those aged $\geq 55$ years the incidence of ischemic stroke was lowest in PrEVISTA (52), 60.3 in NEMESIS and greatest in MSIS (164.3). The incidence of hemorrhagic strokes also varied among cohorts, with the highest rate of subarachnoid hemorrhage in those aged less than 55

years in NEMESIS (Figure 3). In total, MSIS had a greater proportion of strokes occurring in people aged less than 55 years (24\%) than either PrEViSTA (14.7\%) or NEMESIS (10.1\%).

\section{Etiology of stroke}

Stroke type varied according to age, region, and sex. Ischemic stroke was the leading type of stroke among all regions (PrEViSTA:75\%; NEMESIS:70\%; MSIS:82\%; pooled: 75\%). Hemorrhagic stroke accounted for $18 \%$ of strokes and undefined stroke for $5 \%$ of strokes (online supplementary figure I; please see http://stroke.ahajournals.org). 
In those with ischemic stroke, stroke of undetermined source was the most frequent stroke subtype with the greatest incidence rate, followed by embolic stroke in NEMESIS, and small artery disease in both PrEViSTA and MSIS (Table 2, Figure 3) (online supplementary figure I; please see http://stroke.ahajournals.org).In NEMESIS, there was a significantly greater proportion of embolic strokes $(26.30 \%)$ compared to PrEViSTA $(20.70 \%)$ or MSIS (15.20\%; $\mathrm{p}<0.001)$, but these embolic strokes occurred at a much older age in NEMESIS (73.91 \pm 14.46$)$ than in MSIS $(64.57 \pm 14.80 ; \mathrm{p}<0.00)$.

Large artery atherosclerosis was more frequently seen after age 65 years and observed at a similar mean age in the three cohorts $(\mathrm{p}=0.13)$. Small artery disease occurred at younger ages in the MSIS than in the other two cohorts $(\mathrm{p}=0.002)$.

\section{Age pattern of stroke subtypes}

Using SPC analysis we found that first ever strokes occurred most commonly in ages from the early 70s to mid 80s (MSIS: 72-76 years; NEMESIS: 72-86 years; PrEViSTA: 72, 80-84 years) (Figure 4) (online supplementary table II; please see http://stroke.ahajournals.org). In Iran, another peak of first ever stroke was observed in those younger than 44 years of age; these were associated with embolic stroke and stroke due to other rare or uncommon causes. Large artery atherosclerosis was seen frequently in participants in their 70s in all regions. Hemorrhagic stroke showed a different pattern with a relatively younger age band of intracerebral hemorrhage as compared to ischemic stroke in Argentina

and Iran. Subarachnoid hemorrhage was frequently observed in those aged less than 44 years (online supplementary table II; please see http://stroke.ahajournals.org). 


\section{Risk factors}

The prevalence of major risk factors differed in each cohort. Hypertension and diabetes mellitus were the most common risk factors in all countries. While previous TIA, atrial fibrillation and myocardial infarction were more frequently observed in Australia than in the other countries, hypertension and diabetes were more common in Argentina and Iran than in Australia (Table 1). Although smoking was not significantly different among centers, it was more common in men (19.4\%) than women (12.4\%; $\mathrm{p}<0.001$ ) and smokers had their strokes at younger ages (mean age 62.34 \pm 14.09 years) than nonsmokers (mean age $71.80 \pm 14.7$ years; $\mathrm{p}<0.001$ ). Australians had the highest rate of alcohol consumption, particularly in those aged < 55 years (online supplementary figure II; please see http://stroke.ahajournals.org) A history of illicit drug use was reported in 78 (12.5\%) of the MSIS participants; this information was not available for the NEMESIS and PrEViSTA cohorts.

\section{Discussion:}

ASSESS is an ongoing pooled data study that can provide important knowledge about the age, sex, and setting of stroke in different countries, narrowing down the etiological possibilities. This study can also provide the opportunity to assess major questions in stroke, from basic incidence/long-term outcome trends in different countries to preventive and therapeutic measures. Such data are important for both health policy makers, at national and international levels, and stroke epidemiologists.

In this study of pooled population-based data from Argentina, Australia and Iran, we found significant regional variations in stroke incidence, subtypes and risk factors. Importantly, the detailed pooled epidemiologic and clinical information available through the ASSESS study provides the opportunity to determine not only the differences between regions but also to evaluate the possible causes of such 
findings. Our findings are consistent with other studies of global variations in stroke etiology, incidence, and long-term outcomes $(7,16,17)$ as well as variations by factors such as ethnicity and by regions (e.g. stroke belts) within countries. (18) The differences among countries in the age adjusted incidence of stroke can be partially explained by differences in risk factor profiles. A high rate of small artery disease along with a relatively younger age at stroke onset in Argentina and Iran can be attributed to a relatively higher proportion of traditional risk factors, namely hypertension and diabetes mellitus, compared to that seen in Australia. We also observed a greater rate of embolic stroke in Australians as compared to Iranians and Argentinians, which may be due to an increasing prevalence of atrial fibrillation with advancing age. $(13,14)$ In contrast, we observed an unusual peak of ischemic and embolic stroke in Iranians below the age of 44 years which might be related to a greater frequency of valvular heart disease among young Iranians (online supplementary figure II; please see http://stroke.ahajournals.org).

For primary stroke prevention at a worldwide level, we strongly recommend that low- to middle-income countries allocate more resources to control modifiable risk factors, such as hypertension and diabetes. In high income-countries, despite recent temporal declines in the incidence of stroke, (1) the increasing age of the population may lead to another tsunami of stroke. Therefore, detection and treatment of modifiable risk factors, particularly atrial fibrillation, is needed in these countries too, as well as planning for healthcare facilities and medical care for the elderly.

Although we found that only $1.7 \%$ of strokes were attributable to rare and uncommon causes, these are important as they usually affect younger individuals, and thus increase disability adjusted life years in the total population. In addition, this stroke subtype can be different between the regions. For example, 
investigators have previously documented a high rate of sinus thrombosis as a cause of stroke in young adult women in Mashhad, Iran, which might be related to frequent usage of high dose oral contraceptive pills. (19)

Our study has some limitations. In comparison to systematic review and meta-analysis, pooled data studies are more expensive and time-consuming. Although systematic reviews and meta-analyses can combine available data, it is difficult to control for potential confounding factors and adjust information according to study design, data heterogeneity, data collection methods and definition of variables. (20) Furthermore, publication bias is a major limitation of such studies, leading to the overestimation of effect sizes. (21) When compared to systematic reviews and meta-analyses, use of pooled data from many sources, similar to the ASSESS data set, can reduce the chance of publication bias, and can unify variable definitions across different data sets. (22) While the strict inclusion/ exclusion criteria in multicenter clinical trials provide a homogenous group of individuals for data analysis, they are usually limited to an unrepresentative sample of patients with stroke. The heterogeneity of epidemiological studies, particularly population-based samples should not be seen as a weakness but should be utilized to better elucidate differing patterns of stroke between regions. Finally, it is important to consider dynamic changes in stroke trends during the previous decades, in both low- and high-income regions. By collaborating with most recent studies, we can closely monitor such a dynamic trend. For example, although NEMESIS was completed in 1999, results from the more recent Adelaide Stroke Incidence Study showed a similar incidence trend with a similarly large share of cardioembolic strokes. (23) Within the countries, we used stroke information based on single city populations which may not be representative of the whole country. The number of tests undertaken to identify individuals with each TOAST subtype differed both within regions and between regions, and so this somewhat limits the 
interpretation of the data on ischemic stroke subtypes. However, the large proportion of people with neuroimaging studies, including brain CT/ MRIadds credence to the findings. In addition, not all variables were available in all datasets. For example, data on hyperlipidemia and congestive heart failure were not available in NEMESIS, patient occupation was not available in PrEViSTA, and quantification of alcohol consumption was not available in MSIS. Furthermore, although we have data on the presence of risk factors, we do know whether or not these risk factors were controlled. There may be ascertainment bias depending on which hospitals patients attended, or whether they even presented at all. However, PrEViSTA, NEMESIS and MSIS are large epidemiological populationbased studies in three major regions in South America, Australia and Middle East, representing a large community level sample (online Supplementary table III-V; please see http://stroke.ahajournals.org). In addition, having access to all variables in a harmonized data set provided adequate power for detailed statistical analyses.

In summary, we have initiated an ongoing collaboration to pool data from different countries. We welcome other researchers/groups from different settings to participate in this study by providing data, or by proposing projects of national or international interest. We anticipate that this project will yield results of importance to policy makers in different parts of worlds and will provide opportunities for international collaboration.

Acknowledgments: The PrEViSTA was funded by grants from Genzyme Argentina and LePetit Pharma, Laboratorio Roche SAQeI and Boehringer Ingelheim. The NEMESIS was supported by grants from the National Health \& medical Research Council (NHMRC; 154600, 307900, 526601), 
VicHealth, the Foundation for High Blood Pressure Research, and the National Stroke Foundation. The MSIS was supported by a grant from the Mashhad University of Medical Sciences. AGT was supported by a fellowship from the NHMRC (1042600). None of the funders contributed to the current paper.

Source of funding: This research did not receive any specific grant from funding agencies in the public, commercial, or not-for-profit sectors.

Conflict of Interest: The authors have no conflicts of interest 
Table 1. The proportion of vascular risk factors among patients with first-ever strokes.

\begin{tabular}{|c|c|c|c|c|}
\hline Variables & $\begin{array}{c}\text { PrEViSTA } \\
\text { n }=334\end{array}$ & $\begin{array}{c}\text { NEMESIS } \\
\text { n=1316 }\end{array}$ & $\begin{array}{l}\text { MSIS } \\
\mathrm{n}=624\end{array}$ & $\mathrm{P}$ value \\
\hline Age (mean +/- SD) & $70.9(15.0)$ & $73.9(14.5)$ & $64.5(14.8)$ & $<0.001$ \\
\hline Sex: Female, n (\%) & $175(52.4)$ & $731(55.5)$ & 297 (47.6) & 0.005 \\
\hline Hypertension, n (\%) & $263(79.2)$ & $703(54.4)$ & $373(60.2)$ & $<0.001$ \\
\hline Atrial fibrillation, $\mathrm{n}(\%)$ & $54(16.2)$ & $286(21.7)$ & $52(8.60)$ & $<0.001$ \\
\hline Diabetes, n (\%) & $59(17.7)$ & $228(17.5)$ & $187(30.1)$ & $<0.001$ \\
\hline Current smoking & $43(12.9)$ & $188(16.7)$ & $98(15.6)$ & 0.23 \\
\hline Hyperlipidemia, n (\%) & $77(23.10)$ & NA & $148(24.0)$ & 0.80 \\
\hline Alcohol consumption, n (\%) & $20(6.0)$ & $618(57.6)$ & $18(2.9)$ & $<0.001$ \\
\hline Rheumatic heart disease, $\mathrm{n}(\%)$ & NA & $9(0.7)$ & $16(2.6)$ & 0.001 \\
\hline Other valvular heart disease, $\mathrm{n}(\%)$ & NA & $32(2.5)$ & $7(1.1)$ & 0.05 \\
\hline Transient ischemic attack, $\mathrm{n}(\%)$ & $18(5.4)$ & $399(30.3)$ & $49(7.9)$ & $<0.001$ \\
\hline Previous myocardial infarction, $\mathrm{n}(\%)$ & $18(5.4)$ & $174(13.4)$ & $67(10.7)$ & $<0.001$ \\
\hline
\end{tabular}

Abbreviations: PrEVISTA, Program for the Epidemiological Evaluation of Stroke in Tandil, Argentina; NEMESIS, North East MElbourne Stroke Incidence Study; MSIS, Mashhad Stroke Incidence Study. 
Table 2. The proportion and annual age-adjusted incidence per 100,000 of stroke types.

Proportion of stroke subtypes

$$
\mathrm{n}(\%)
$$

\section{Stroke type and subtypes}

Age adjusted incidence rate per

100,000

\section{PrEViSTA NEMESIS MSIS P value}

PrEViSTA NEMESIS MSIS

\section{Ischemic stroke}

Embolic stroke

Large artery

atherosclerosis

Small vessel disease

Stroke of undetermined

source

Stroke of other determined etiologies

\section{Hemorrhagic stroke}

Subarachnoid hemorrhage

Intracerebral hemorrhage

Undefined Stroke

$$
\text { 251(75.1) }
$$

$921(70)$

$512(82.1)$

$<0.001$

$52(20.8) \quad 259(26.3) \quad 77(15.2) \quad<0.001$

$42(16.8)$

$190(19.3) \quad 72(14.3)$

0.03

11.2

64.8

70.1

194.4

12.2

16.2

27.8

$72(28.8)$

$150(15.3)$

$114(22.2)$

$<0.001$

20.4

10.9

43.2

$84(33.6) \quad 369(37.5) \quad 225(43.9) \quad 0.01$

20.9

25.4

89.3

$14.9 \quad 28.2$ 
NA, No data available; PrEVISTA, Program for the Epidemiological Evaluation of Stroke in Tandil, Argentina; NEMESIS, North East MElbourne Stroke Incidence Study; MSIS, Mashhad Stroke Incidence Study. 


\section{Figure 1. ASSESS centers: Current collaborative centers in ASSESS}

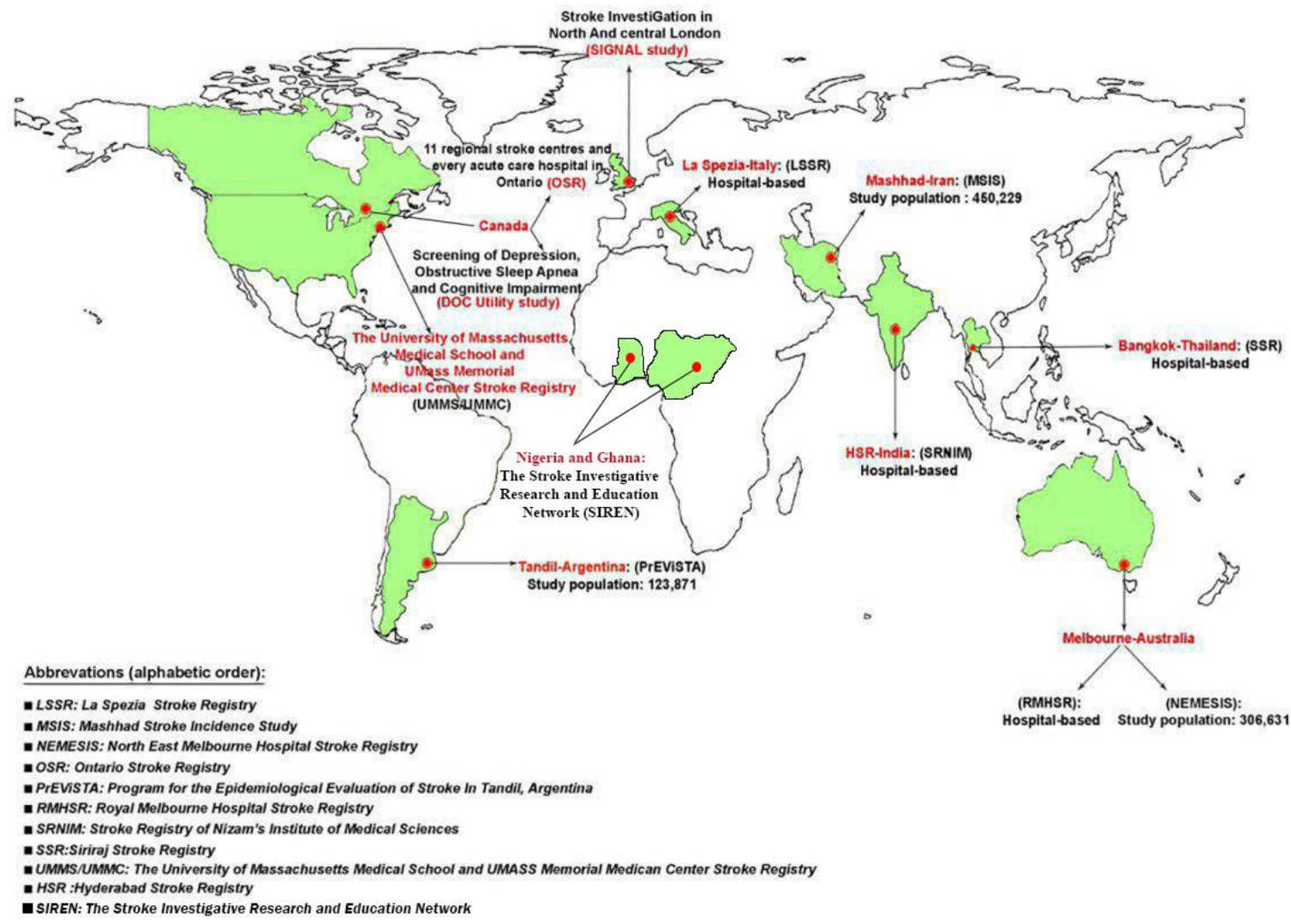


Figure-2: Age pyramids of all patient with first-ever stroke.

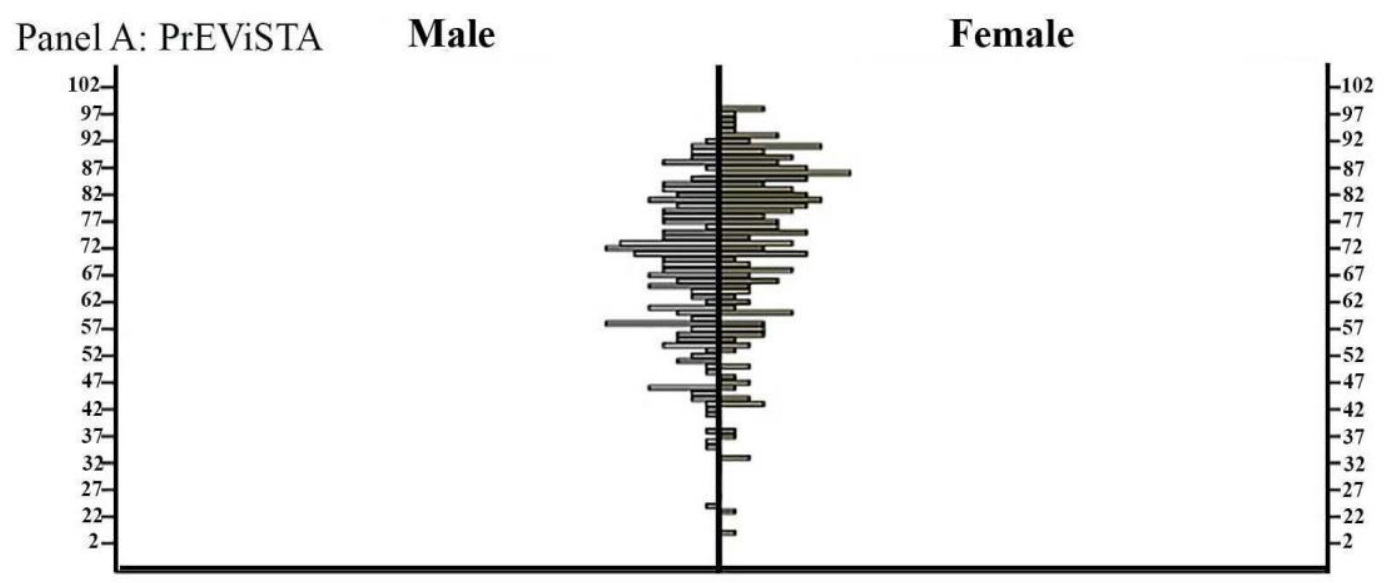

Panel B: NEMESIS

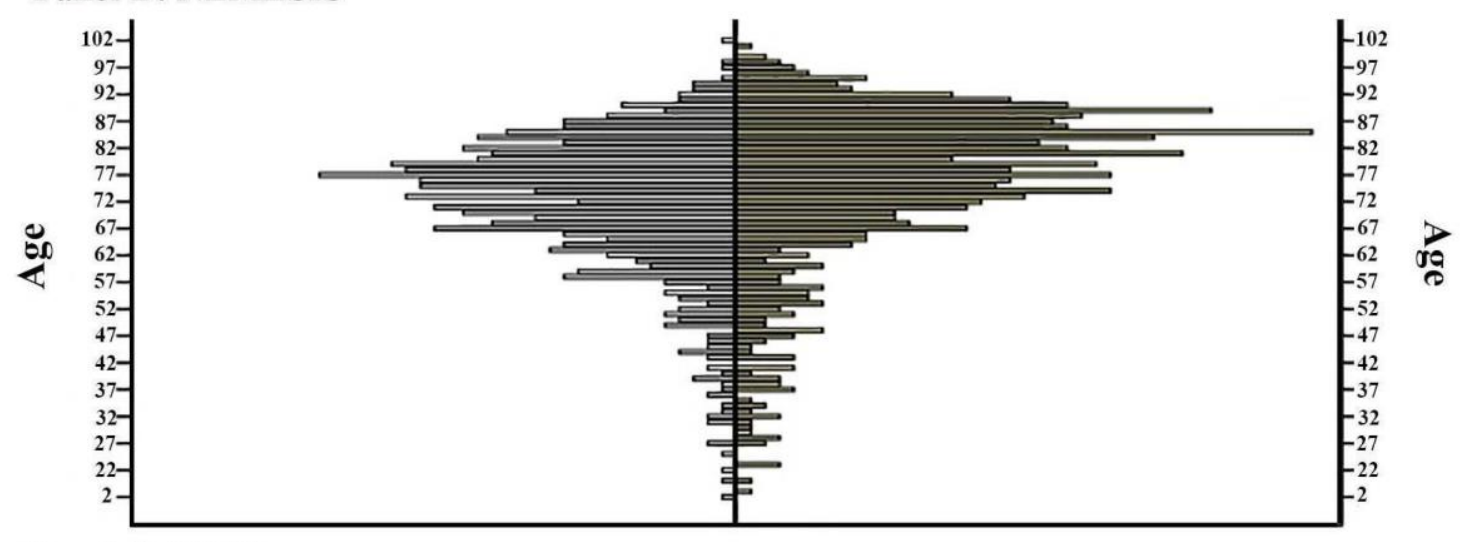

Panel C: MSIS

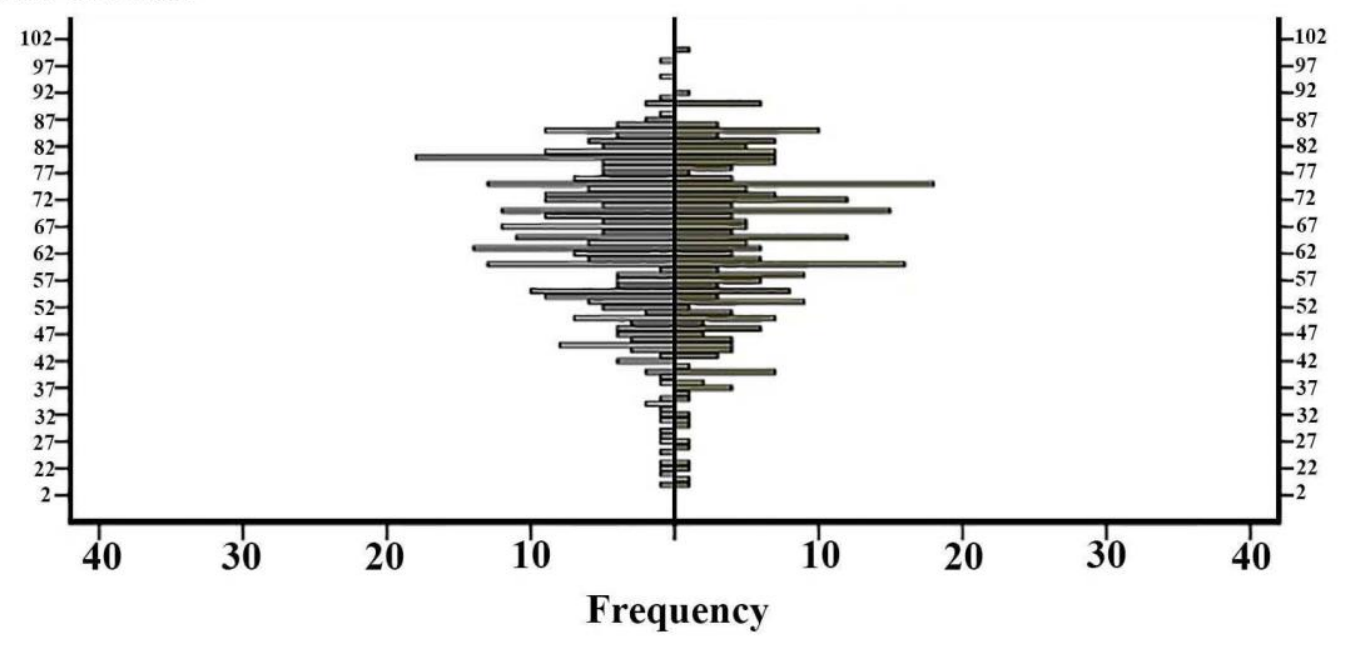


Figure 3. The WHO age-adjusted incidence rates (per 100,000) for first-ever strokes according to their subtypes.

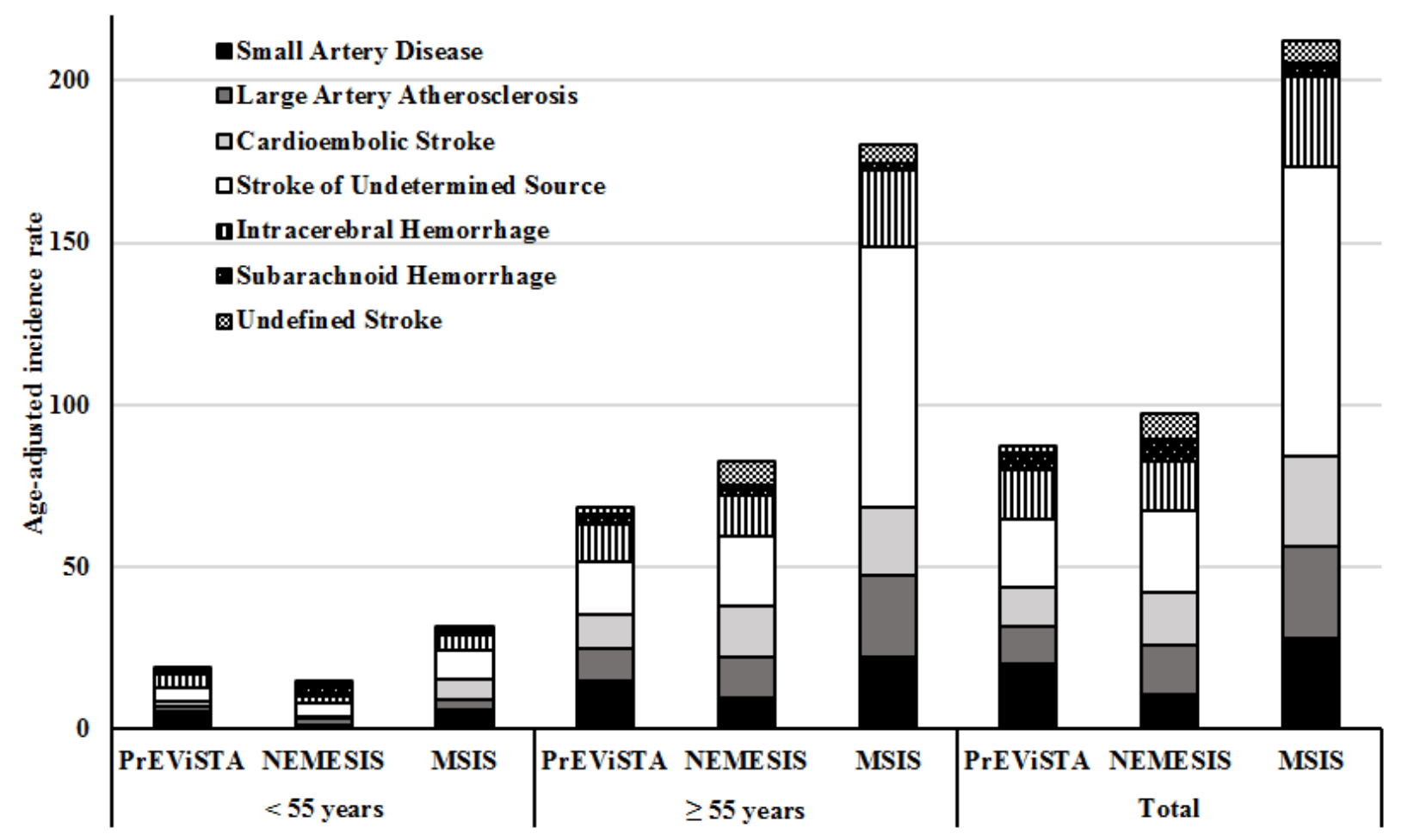


Figure 4. The statistical Process Control (SPC) chart, categorizing the ages with the highest/lowest frequency of first-ever stroke.
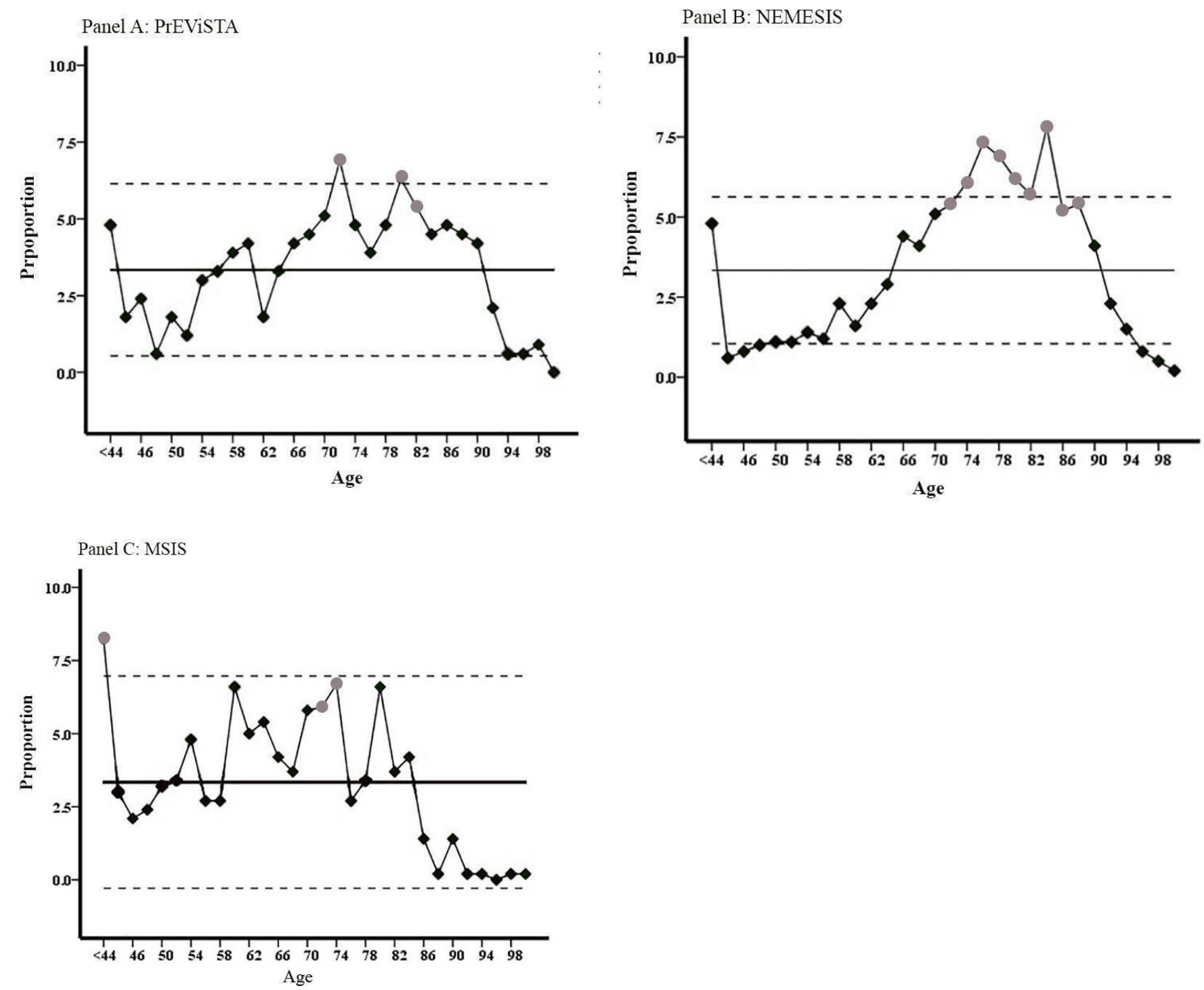

Over expected proportion of first-ever stroke in defined age groups

Expected proportion of first-ever stroke in defined age groups 


\section{References:}

1. Feigin VL, Forouzanfar MH, Krishnamurthi R, Mensah GA, Connor M, Bennett DA, et al. Global and regional burden of stroke during 1990-2010: findings from the Global Burden of Disease Study 2010. Lancet. 2014;383:245-54.

2. Hachinski V, Azarpazhooh MR. Stroke is a burdensome but preventable brain disorder. Lancet Neurol. 2016;15:892-3.

3. Bahit MC, Coppola ML, Riccio PM, Cipriano LE, Roth GA, Lopes RD, et al. First-Ever Stroke and Transient Ischemic Attack Incidence and 30-Day Case-Fatality Rates in a Population-Based Study in Argentina. Stroke 2016;47:1640-2.

4. Thrift AG, Dewey HM, Macdonell RA, McNeil JJ, Donnan GA. Stroke incidence on the east coast of Australia: the North East Melbourne Stroke Incidence Study (NEMESIS). Stroke. 2000;31:2087-92.

5. Thrift AG, Dewey HM, Sturm JW, Srikanth VK, Gilligan AK, Gall SL, et al. Incidence of stroke subtypes in the North East Melbourne Stroke Incidence Study (NEMESIS): differences between men and women. Neuroepidemiology. 2009;32:11-8.

6. Azarpazhooh MR, Etemadi MM, Donnan GA, Mokhber N, Majdi MR, Ghayour-Mobarhan M, et al. Excessive incidence of stroke in Iran: evidence from the Mashhad Stroke Incidence Study (MSIS), a population-based study of stroke in the Middle East. Stroke. 2010;41:e3-10.

7. Saber H, Thrift AG, Kapral MK, Shoamanesh A, Amiri A, Farzadfard MT, et al. Incidence, recurrence, and long-term survival of ischemic stroke subtypes: A population-based study in the Middle East. Int J Stroke : Off J Int Stroke Soc. 2017;12:835-43.

8. Kaul S, Sunitha P, Suvarna A, Meena AK, Uma M, Reddy JM. Subtypes of Ischemic Stroke in a Metropolitan City of South India (One year data from a hospital based stroke registry). Neurol 
India. 2002;50 Suppl:S8-14.

9. Akpalu A, Sarfo FS, Ovbiagele B, Akinyemi R, Gebregziabher M, Obiako R, et al. Phenotyping Stroke in Sub-Saharan Africa: Stroke Investigative Research and Education Network (SIREN) Phenomics Protocol. Neuroepidemiology. 2015;45:73-82.

10. Morris K. Charles Rotimi: engaging Africa in human genomic research. Lancet. 2010;376:1383.

11. H3Africa Consortium, Rotimi C, Abayomi A, Abimiku A, Adabayeri VM, Adebamowo C, et al. Research capacity. Enabling the genomic revolution in Africa. Sci. 2014 Jun 20;344:1346-8.

12. Rotimi CN, Jorde LB. Ancestry and disease in the age of genomic medicine. New Engl J Med. 2010;363:1551-8.

13. Owolabi M, Ogbole G, Akinyemi R, Salaam K, Akpa O, Mongkolwat P, et al. Development and Reliability of a User-Friendly Multicenter Phenotyping Application for Hemorrhagic and Ischemic Stroke. J Stroke Cerebrovasc Dis : Off J Natl Stroke Assoc. 2017;26:2662-70.

14. Cher DJ, Deubner DC, Kelsh MA, Chapman PS, Ray RM. Assessment of the beryllium lymphocyte proliferation test using statistical process control. Inhal Toxicol]. 2006;18:901-10.

15. Carey RG. How Do You Know That Your Care Is Improving? Part I: Basic Concepts in Statistical Thinking. J Ambul Care Manag. 2002;25:80-87.

16. Gandolfo C, Conti M. Stroke in young adults: epidemiology. Neurol Sci : Off J Ital Neurol Soc Ital Soc Clin Neurophysiol. 2003;24 Suppl 1:S1-3.

17. Medin J, Nordlund A, Ekberg K, Swedish Hospital Discharge Register. Increasing stroke incidence in Sweden between 1989 and 2000 among persons aged 30 to 65 years: evidence from the Swedish Hospital Discharge Register. Stroke. 2004;35:1047-51.

18. Ismailov RM. The role of erythropoietin in the "stroke belt" phenomenon. Med hypotheses. 2012;79:181-5. 
19. Sasannejad P, Mellat Ardekani A, Velayati A, Shoeibi A, Saeidi M, Foroughipour M, et al. Cerebral vein thrombosis in women using short course oral contraceptive consumption. Iran $\mathbf{J}$ Reprod Med. 2012;10:537-42.

20. Shapiro S. Meta-analysis/Shmeta-analysis. Am J Epidemiol. 1994;140:771-8.

21. Blettner M, Sauerbrei W, Schlehofer B, Scheuchenpflug T, Friedenreich C. Traditional reviews, meta-analyses and pooled analyses in epidemiology. Int J Epidemiol. 1999;28:1-9.

22. Collaborative Group on Hormonal Factors in Breast Cancer. Breast cancer and hormonal contraceptives: collaborative reanalysis of individual data on 53297 women with breast cancer and 100239 women without breast cancer from 54 epidemiological studies. Lancet. $1996 ; 347: 1713-27$.

23. Leyden JM, Kleinig TJ, Newbury J, Castle S, Cranefield J, Anderson CS, et al. Adelaide stroke incidence study: declining stroke rates but many preventable cardioembolic strokes. Stroke. $2013 ; 44: 1226-31$. 